University of Wollongong

Research Online

Faculty of Social Sciences - Papers (Archive) Faculty of Arts, Social Sciences \& Humanities

2015

Gross morphological brain changes with chronic, heavy cannabis use

Valentina Lorenzetti

University of Melbourne

Nadia Solowij

University of Wollongong, nadia@uow.edu.au

Sarah Whittle

University of Melbourne

Alex Fornito

University of Melbourne

Daniel Lubman

Monash University

See next page for additional authors

Follow this and additional works at: https://ro.uow.edu.au/sspapers

Part of the Education Commons, and the Social and Behavioral Sciences Commons

Research Online is the open access institutional repository for the University of Wollongong. For further information contact the UOW Library: research-pubs@uow.edu.au 


\title{
Gross morphological brain changes with chronic, heavy cannabis use
}

\begin{abstract}
We investigated the morphology of multiple brain regions in a rare sample of 15 very heavy cannabis users with minimal psychiatric comorbidity or significant exposure to other substances (compared with 15 age- and IQ-matched non-cannabis-using controls) using manual techniques. Heavy cannabis users demonstrated smaller hippocampus and amygdala volumes, but no alterations of the orbitofrontal and anterior- and paracingulate cortices, or the pituitary gland. These findings indicate that chronic cannabis use has a selective and detrimental impact on the morphology of the mediotemporal lobe.
\end{abstract}

\section{Keywords}

heavy, chronic, cannabis, changes, gross, brain, morphological

Disciplines

Education | Social and Behavioral Sciences

\section{Publication Details}

Lorenzetti, V., Solowij, N., Whittle, S., Fornito, A., Lubman, D. I., Pantelis, C. \& Yücel, M. (2015). Gross morphological brain changes with chronic, heavy cannabis use. British Journal of Psychiatry, 206 (1), 77-78.

\section{Authors}

Valentina Lorenzetti, Nadia Solowij, Sarah Whittle, Alex Fornito, Daniel Lubman, Christos Pantelis, and Murat Yücel 


\section{Gross morphological changes with chronic, heavy cannabis use}

Valentina Lorenzetti, Ph.D. a, b, Nadia Solowij, Ph.D. c, Sarah Whittle Ph.D. b, d, Alex Fornito, Ph.D.a, b, Dan I. Lubman, PhD, F.A.Ch.A.M., F.R.A.N.Z.C.P.e, Christos Pantelis, M.D., M.R.C.Psych., F.R.A.N.Z.C.P. ${ }^{\mathrm{b}}$ Murat Yücel, Ph.D., M.A.P.S.a,b*

a. Monash Clinical and Imaging Neuroscience, Monash University, VIC Australia

b. Melbourne Neuropsychiatry Centre, Department of Psychiatry, The University of Melbourne and Melbourne Health, VIC Australia

c. School of Psychology, University of Wollongong, NSW Australia

d. Department of Psychology, The University of Melbourne, Australia

e. Turning Point, Eastern Health and Monash University, VIC Australia

Declaration of interest: None

Funding: This study was supported by grants from the Clive and Vera Ramaciotti Foundation, the Schizophrenia Research Institute using infrastructure funding from NSW Health, the University of Wollongong and the Australian Research Council Future Fellowship (FT110100752) (Associate Professor Solowij), the Melbourne Neuroscience Institute Postdoctoral Fellowship, The University of Melbourne (Dr Lorenzetti); NHMRC Career Development Fellowship (Dr Whittle); the Monash Larkins Fellowship (Associate Professor Fornito); the NHMRC Senior Principal Research Fellowship (ID: 628386) and NARSAD Distinguished Investigator Award (Professor Pantelis) and the NHMRC Senior Research Fellowship (Professor Yücel).

Corresponding author:

* Professor Murat Yücel

Monash Clinical and Imaging Neuroscience $1^{\text {st }}$ floor, Monash Biomedical Imaging 770 Blackburn Road, Clayton, Victoria 3800, Australia Phone: +61(3) 9902-9765 Email: $\underline{\text { murat.yucel@monash.edu }}$ 
British Journal of Psychiatry 2015, 206, 77-78.

Running Title: Gross morphological changes in cannabis users 


\begin{abstract}
We investigated the morphology of multiple brain regions in a rare sample of 15 very heavy cannabis users with minimal psychiatric comorbidity or significant exposure to other substances (compared with 15 age- and IQ-matched non-cannabis-using controls) using manual techniques. Heavy cannabis users demonstrated smaller hippocampus and amygdala volumes, but no alterations of the orbitofrontal and anterior- and paracingulate cortices, or the pituitary gland. These findings indicate that chronic cannabis use has a selective and detrimental impact on the morphology of the mediotemporal lobe.
\end{abstract}

Key Words: MRI, cannabis, brain, morphology 


\section{Introduction}

There is growing evidence that long-term heavy cannabis use is associated with anatomical alterations across multiple brain regions (1). Many studies employing structural Magnetic Resonance Imaging (MRI) techniques have adopted automated parcellation techniques, which can be problematic when measuring brain areas that are highly variable across individuals and where the boundaries are not clear $(\underline{2}, \underline{3})$. Our group has previously used manual tracing techniques, which are sensitive to inter-individual neuroanatomical variability, to identify gross abnormalities in hippocampal and amygdala morphology in long-term heavy cannabis users (뜨). Whether these abnormalities occur in other brain areas is yet to be elucidated. Here, we investigate volumetric alterations of key brain regions that may be impacted by long-term heavy cannabis use, namely the orbitofrontal and anterior cingulate cortices, as well as the pituitary gland, all of which are rich in cannabinoid receptors and are involved in cognition and the regulation of emotion and stress levels, the alteration of which is associated with cannabis exposure $(\underline{5}, \underline{6})$. We report these data alongside our previously reported hippocampus and amygdala volumes in the same sample for comparative purposes ( $\underline{4})$.

\section{Method}

We recruited 15 male long-term heavy cannabis users with the highest level of cumulative cannabis exposure of all samples examined to date (21 years of regular use and lifetime

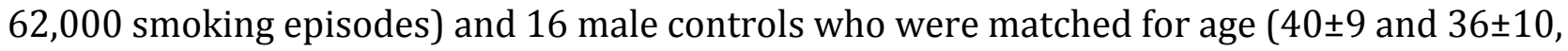
respectively), intelligence quotient (109 \pm 6 and $114 \pm 8$, respectively) and education years $(13 \pm 3$ and $15 \pm 4$, respectively ( $\underline{4})$. Participants had very limited exposure to substances other than cannabis (<10 episodes) and no history of medical, neurologic, or psychiatric conditions. Cannabis users were smoking at a rate of $28 \pm 3$ days per month and were consuming approximately 212 joints per month. Structural T1 weighted MRI images were acquired with a 3T Phillips Intera scanner (Symbion Clinical Research Imaging Centre, Prince of Wales Medical Research Institute, Sydney, Australia), matrix size 256x256x180, with a isotropic voxel size of $1 \mathrm{~mm}^{3}$. 
All regions of interest were manually delineated using ANALYZE 11.0 software. We examined the anatomy of functionally and anatomically distinct components for the orbitofrontal cortex (i.e., medial and lateral sub-regions), anterior cingulate and paracingulate cortices, both of which were parceled into dorsal, rostral and ventral portions ( $\underline{3})$. Inter and intra-rater reliabilities for manual tracings were assessed by intra-class correlation coefficients. The range was .80-.98, indicating that the implemented tracing methods were reliable means to measure brain morphology, while considering interindividual variations in sulcal and gyral anatomy.

The impact of cannabis use on regional brain volumes was examined by performing separate ANOVAs on each primary brain region, with hemisphere (i.e., left or right) as repeated measure, group as between-subjects factor and regional brain volumes as dependent variables. For the orbitofrontal cortex, region (i.e., lateral or medial) was entered as an additional within-subjects factor. For the anterior cingulate cortex, region (dorsal, rostral, ventral) and cortex (cingulate and para-cingulate cortex,) were entered as an additional within-subjects factor. Hemisphere was not included in the analysis of pituitary volume. Main effects and interactions were evaluated using Greenhouse-Geisser corrected degrees of freedom with $\mathrm{a}=0.0125$ ( 0.05 divided by the number of brain regions investigated). Significant effects were further investigated with post-hoc pairwise contrasts evaluated against a Bonferroni-adjusted a to correct for multiple comparisons. Statistical analyses were performed using SPSS version 20.0.

\section{Results}

Cannabis users, compared to controls, demonstrated reduced hippocampal and amygdala volumes, as previously reported ( $\underline{4}$ ), but no alteration of the orbitofrontal and anterior cingulate cortices, or the pituitary gland (Figure 1). There was no significant interaction between group and hemisphere on the examined brain regions. 
Figure 1: Regional brain volumes in heavy cannabis users (white) and non-cannabis using controls (black).

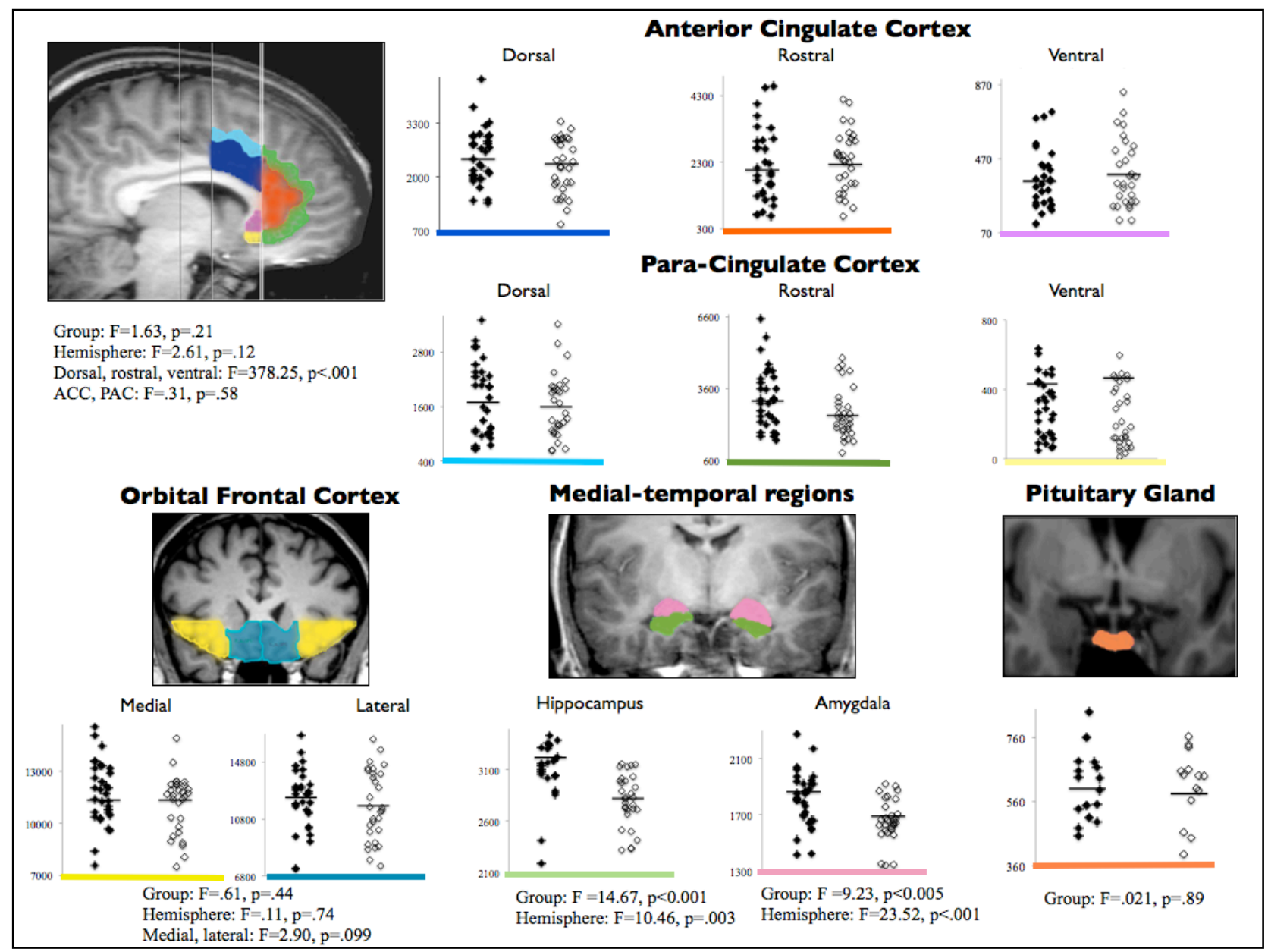

For all comparisons, $\mathrm{df}=1,29$. 


\section{Discussion}

Our findings suggest that heavy cannabis use is associated with localised neuroanatomical reductions in medial-temporal regions, largely hippocampal, with clear divergence between groups in distribution of hippocampal volumetric data relative to significant overlap for other regions. We failed to find any gross alteration in the orbitofrontal cortex, anterior cingulate cortex and pituitary gland. Our results accord with other studies showing effects of heavy cannabis use on medial-temporal (largely hippocampal) regions (ㄱ), but are the only findings to date showing volumetric reductions in the amygdala, as previously reported ( $\underline{4})$. Our results contrast however, with previously reported prefrontal alterations in cannabis users who were smoking at lower rates than those of our present study (1). Thus, heavy exposure to cannabis may adversely impact the hippocampus, while prolonged exposure may be required for other brain regions (e.g., prefrontal cortex). Alternatively, the small size of the investigated sample may also explain the lack of a detectable effect in these regions.

Although the small sample size and the distinct characteristics of our sample (i.e., no significant exposure to substances other than cannabis and no comorbid psychopathology) may limit the generalisability of our findings, this study offers a relatively pure insight into the neurobiological sequalae of prolonged, heavy cannabis use on multiple brain regions. Our findings support the notion that chronically high cannabinoid exposure has a selective and detrimental impact on medial-temporal brain regions. Volumetric reductions of these regions may have functional significance ( $\underline{8})$ and have been previously associated with heightened subclinical psychotic symptoms in this sample (4). Combined multimodal neuroimaging techniques should be used in future studies to elicit more comprehensive information on the neurobiology of long-term heavy cannabis use, and to elucidate the pathophysiological mechanisms involving metabolic processes, functional and structural connectivity in brain regions that are high in cannabinoid receptors and may be vulnerable to the adverse impact of long-term heavy cannabis exposure $(\underline{9}, \underline{10})$.

\section{References}


1. Lorenzetti V, Solowij N, Fornito A, Lubman D, Yucel M. The Association between Regular Cannabis Exposure and Alterations of Human Brain Morphology: An Updated Review of the Literature. Curr Pharm Des. 2013.

2. Morey RA, Petty CM, Xu Y, Hayes JP, Wagner HR, 2nd, Lewis DV, et al. A comparison of automated segmentation and manual tracing for quantifying hippocampal and amygdala volumes. NeuroImage. 2009; 45(3): 855-66.

3. Fornito A, Whittle S, Wood S, Velakoulis D, Pantelis C, Yücel M. The Influence of Sulcal Variability on Morphometry of the Human Anterior Cingulate and Paracingulate Cortex. NeuroImage. 2006; 33: 843 - 54.

4. Yücel M, Solowij N, Respondek C, Whittle S, Fornito A, Pantelis C, et al. Regional brain abnormalities associated with heavy long-term cannabis use. Arch Gen Psychiatry. 2008; 65(6): 1 - 8 .

5. Landfield PW, Caldwallader LB, Vinsant S. Quantitative changes in hippocampal structure following long-term exposure to $\Delta$ 9-tetrahydrocannabinol: possible mediation by glucocorticoid systems. Brain Research. 1988; 443(1 - 2): 47 - 62.

6. Cheetham A, Allen NB, Whittle S, Simmons J, Yücel M, Lubman DI. Orbitofrontal Volumes in Early Adolescence Predict Initiation of Cannabis Use: A 4-Year Longitudinal and Prospective Study. Biol Psychiatry. 2011.

7. Rocchetti M, Crescini A, Borgwardt S, Caverzasi E, Politi P, Atakan Z, et al. Is cannabis neurotoxic for the healthy brain? A meta-analytical review of structural brain alterations in non-psychotic users. Psychiatry Clin Neurosci. 2013; 67(7): 483-92.

8. Hickie I, Naismith S, Ward PB, Turner K, Scott E, Mitchell P, et al. Reduced hippocampal volumes and memory loss in patients with early- and late-onset depression. The British Journal of Psychiatry. 2005; 186: 197-202.

9. Harding IH, Solowij N, Harrison BJ, Takagi M, Lorenzetti V, Lubman DI, et al. Functional connectivity in brain networks underlying cognitive control in chronic cannabis users. Neuropsychopharmacology. 2012; 37(8): 1923-33. 
10. Zalesky A, Solowij N, Yucel M, Lubman DI, Takagi M, Harding IH, et al. Effect of longterm cannabis use on axonal fibre connectivity. Brain. 2012; 135(Pt 7): 2245-55. 